Michael Brzoska

\title{
Friedensforschung und Internationale Beziehungen - Lob der Verschiedenheit
}

\begin{abstract}
Friedensforschung und Internationale Beziehungen (IB) sind - in unterschiedlicher Intensität und in verschiedenen Formen - seit einem halben Jahrhundert eng miteinander verbunden. Friedensforschung ist nicht denkbar ohne IB, sowohl in ihrer historischen Entwicklung als auch gegenwärtig. Friedensforschung hat sich als Kritik herrschender Doktrinen der IB entwickelt. Viele der in den IB seit den 1990 er Jahren intensiv diskutierten Konzepte sind in der Friedensforschung vorgedacht worden. Die seit den 1990er Jahren festzustellende Professionalisierung der Friedensforschung hat wiederum dazu geführt, dass Forschungsdesigns, exakte Methoden und Theoriebildung aus den IB übernommen wurden. Friedensforschung und IB unterscheiden sich aber weiterhin in wichtigen Punkten, insbesondere Wertbezug, Praxisorientierung und den Konzepten von Interdisziplinarität. Friedensforschung sollte nicht als Disziplin verstanden werden, sondern Ansätze und Methoden aus vielen Disziplinen bündeln, um wissenschaftich fundierte Analysen mit dem Ziel der Verhinderung und Verminderung organisierter Gewalt zu liefern.
\end{abstract}

\section{Der Standort der Friedensforschung}

Friedensforschung und die Forschung $\mathrm{zu}$ internationaler Politik, die unter dem Dach der Internationalen Beziehungen (IB) stehen, sind, wie Harald Müller und Klaus Schlichte in ihren Beiträgen deutlich machen, eng miteinander verbunden. Im letzten halben Jahrhundert - also seit es Friedensforschung gibt - hat es unterschiedliche Phasen der Interaktion gegeben. Es gibt wenig Anlass anzunehmen, dass die beiden Forschungstraditionen zunehmend auseinanderdriften oder - im Gegenkurs - miteinander verschmelzen. Beides wäre auch nicht wünschenswert.

Friedensforschung und IB überlappen sich in vielem, sie unterscheiden sich aber tendenziell in einer Reihe von Dimensionen. »Tendenziell«, weil, wie auch Harald Müller in seinem Beitrag zeigt, eine Zuordnung oft schwierig ist. Wesentliche Unterschiede, die auch in der Literatur immer wieder genannt werden, sind Gegenstandsabgrenzung, Normativität, Praxisorientierung und Interdisziplinarität (Schlotter/Wisotzki 2011: 9-45). Die Positionierungen von Friedensforschung und IB sind hier jeweils - mit vielen Abweichungen in Einzelfällen - im Mittelwert deutlich unterscheidbar. Friedensforschung definiert sich über einen, wenn auch im Einzelnen sehr unterschiedlich abgegrenzten, Forschungsgegenstand, der deutlich stärker eingeengt ist als das Themenfeld der IB. Während Wertebezug in der Friedensforschung von hoher Bedeutung ist, wird normativ begründete Forschung in den IB zumindest überwiegend - abgelehnt. Praxisorientierung hat für die Friedensfor- 
schung einen dominanten strategischen Stellenwert, ein Platz, den in den IB eher die Theoriebildung einnimmt.

Von der Friedensforschung wird zu Recht verlangt, dass sie zu Fragen der Zukunft von Krieg und Frieden arbeitet. Sie muss dabei auf die wissenschaftliche Grundlage ihrer Aussagen bedacht sein. Die IB, zum Beispiel mit ihrer Forschung zu internationalen Machtverschiebungen (Flemes 2010) ist hier ein starker Partner. Gleichzeitig kann die Friedensforschung, anders als die IB, ihren Anspruch, in kritischer Distanz zu herrschenden Strukturen und Erwartungen, Empfehlungen für gesellschaftliches und politisches Handeln zu geben, nicht aufgeben.

In diesem Beitrag möchte ich einige Vor- und Nachteile der Positionierungen der Friedensforschung im Verhältnis zu den IB kommentieren. Abschließend benenne ich einige thematische Schwerpunkte für die Friedensforschung der nächsten Jahre, die aus meiner Sicht besonders wichtig sind.

Friedensforschung steht in diesem Beitrag als Kurzform für Friedens- und Konfliktforschung, die ich wie Schlichte (2012, in diesem Heft) in seinem Beitrag als analytische Einheit sehe. Davon grenze ich die insbesondere im angelsächsischen Raum verbreiteten Friedensstudien mit ihrem Fokus auf Gruppenkonflikte und deren Vermeidung und Verarbeitung (Lopez 1999) sowie den Sammelbegriff Friedenswissenschaften $\mathrm{ab}$, unter dessen Dach häufig alle akademischen Bemühungen versammelt werden, die sich der Beförderung von Frieden widmen (Battke/Hauswedell 1993). Friedensforschung ist historisch und institutionell den IB weit stärker verbunden als die Friedensstudien, in denen Psychologie und Geisteswissenschaften einen hohen Stellenwert haben, und die disziplinär additiven Friedenswissenschaften.

\section{Ein sehr kurzer historischer Rückblick}

Friedensforschung ist nicht denkbar ohne IB, sowohl in ihrer historischen Entwicklung als auch gegenwärtig. Friedensforschung hat sich als Kritik herrschender Doktrinen der IB in den späten 1950er und frühen 1960er Jahren entwickelt (Hauswedell 1997; Wasmuht 1998).

Von vorrangiger Bedeutung war dabei die Abgrenzung von der Kosmologie des Realismus, insbesondere in Form der Strategischen Studien. Die thematischen Gegenstände der frühen Friedensforschung entsprachen weitgehend denjenigen der Strategic Studies - Gewaltkonflikte zwischen großen gesellschaftlichen Gruppen, insbesondere Staaten - aber mit einer völlig anderen Ontologie (siehe Tabelle 1). Es sollte nicht, wie bei den Strategischen Studien, vor allem darum gehen, den Vorteil des Staates oder der Staatengruppe zu befördern, mit der sich die Wissenschaftlerin identifizierte, sondern um Gewaltvermeidung in universaler Perspektive (Buzan/Hansen 2009: 101-155).

Dass Friedensforschung ohne IB weder intellektuell noch institutionell auskommt, ist aber auch eine aktuelle Diagnose: Da wo Friedensforschung in Deutschland betrieben wird, ist sie, wie Harald Müller belegt, mindestens eng verwoben, 
wenn nicht ad personam identisch mit der Forschung zu internationalen Beziehungen. Die überwiegende Zahl der wissenschaftlichen Mitarbeiterinnen an Forschungsinstituten mit Friedensforschung im Titel haben diese fachliche Orientierung.

Umgekehrt war die IB bis in die 1970er Jahre hinein ohne Friedensforschung sehr wohl denkbar. Seitdem hat die Friedensforschung einen starken Einfluss auf die IB, insbesondere in Deutschland, Skandinavien und einigen Ländern des globalen Südens, mit deutlichen Abstrichen auch in Großbritannien und den USA, gehabt. Beiträge und Personen aus der Friedensforschung waren zentral für die Erneuerung der IB seit den 1970er Jahren, sie hat den Paradigmenwechsel in den IB von Spielarten des Realismus zu Ausformungen des Idealismus nachhaltig befördert.

In den IB erfuhren einige aus der Friedensforschung stammende Ideen, Konzepte und Analyseansätze eine deutlich systematischere Behandlung als dies in der Friedensforschung der Fall gewesen war. Zahlreiche Beispiele lassen sich hierfür anführen. So ist das Konzept der »menschlichen Sicherheit» eng verwandt mit Johan Galtungs Kategorisierung von Gewalt als zentraler Kategorie von Friedenspolitik. Das wird insbesondere deutlich, wenn die beiden dominierenden Ausprägungen des Konzeptes, die sich knapp als »japanische« und »kanadische« bezeichnen lassen, der Unterscheidung zwischen direkter und struktureller Gewalt bei Galtung gegenübergestellt werden (Brzoska 2004a: 240-248). In der Friedensforschung ist früh die Bedeutung nicht-staatlicher Akteure für die Gestaltung der internationalen Politik benannt worden, die später in der Forschung zu Nicht-Regierungsorganisationen aufgenommen wurde. Die Feindbildforschung, beliebter Ansatz der Friedensforschung in den 1970er Jahren (Nicklas/Gantzel 1975), lässt sich in methodischer wie erkenntnistheoretischer Sicht als Vorläufer konstruktivistischer Analysen sehen.

In der Friedensforschung wurden zahlreiche Konzepte nach ihrer Einführung oft lediglich heuristisch benutzt, ohne tiefergehende Beschäftigung mit methodischen Fragen oder dem Versuch grundlegender Theoriebildung (aber vgl. etwa Senghaas 1972: 28-93; Czempiel 1981). Die Friedensforschung »professionalisierte« sich allerdings zunehmend parallel zur Erneuerung der IB seit den 1980er Jahren. Durchdachte Forschungsdesigns, exakte Methoden und Theoriebildung wurden zunehmend zum Standard in der Friedensforschung. Friedensforschung und IB näherten sich auf dem Weg der Friedensforschung zu strengeren akademischen Standards und dem der IB zu größerer Offenheit gegenüber neuen Ideen und Ansätzen gegenseitig stark an.

Nicht alle, die sich dem normativen Impetus einer Forschung für den Frieden verpflichtet fühlten, sahen darin einen Fortschritt. Es entwickelte sich eine Kluft zwischen der methodisch zunehmend im sozialwissenschaftlichen Mainstream schwimmenden Friedensforschung und den disziplinär völlig heterogenen Friedenswissenschaften ebenso wie den Friedensstudien, in denen Forschung vorrangig der Entwicklung von sozialen und politischen Handlungskonzepten sowie der teilnehmenden Beobachtung und Evaluation von Aktionen dient (Dietrich et al. 2011: 5-11). Damit akzentuierte sich ein schon in der Entstehungsphase der Friedensforschung sichtbarer Unterschied zwischen der deutschen und skandinavischen Frie- 
densforschung und den »peace studies«, die in Deutschland relativ wenig Fuß gefasst haben, in den USA, aber auch in einigen Ländern des globalen Südens hingegen institutionell besser verankert sind als die Friedensforschung. ${ }^{1}$ Die deutsche Friedensforschung wurde in ihrem akademischen Charakter den IB ähnlicher und grenzte sich zunehmend gegenüber den Peace Studies ab. ${ }^{2}$

Der weiteren Annäherung von Friedensforschung und IB stand in den 1990er Jahren zunächst die wachsende Bedeutung post-positivistischer Ansätze in den IB entgegen. Die Friedensforschung in Deutschland ist nach ihrer eher pragmatistischen Gründungsphase überwiegend Ideen der kritischen Theorie verpflichtet, mit Ausschlägen in Richtung Positivismus sowohl im Bereich marxistisch angehauchter Forschungen als auch bei statistisch-quantitativ ausgerichteten Arbeiten. Ein Forschungsstrang, dessen Orientierung an einer Forschung für den Frieden konstitutiv war, musste mit der post-positivistischen Herausforderung an die Möglichkeit »objektiver« Wahrheiten Schwierigkeiten haben (Bonacker 2011: 46-78).

Allerdings gehören konstruktivistische Ansätze auf post-positivistischer Grundlage inzwischen zum gängigen Repertoire der Friedensforschung. Ein Beispiel hierfür ist das Konzept der Securitization. Ursprünglich von Ole Wæver am Copenhagen Peace Research Institute (COPRI) in Dänemark erarbeitet (Guzzini/Jung 2004), fand es in den IB seit der Mitte der 1990er Jahre starke Beachtung. Autorinnen, die aus der Friedensforschung kommen, tragen zur kaum noch überschaubaren Literatur zu »Versicherheitlichung« bei, wenn auch häufiger als Anwender denn als Weiterentwickler (Buzan/Hansen 2009). Ein Beispiel ist die Diskussion um die »Versicherheitlichung« des Klimawandels (Brzoska et al. 2011).

\section{Gegenstandsbereich und Normativität}

Insbesondere mit Blick auf die Ausweitung der Friedensforschung um post-positivistische Beiträge hat Thorsten Bonacker argumentiert, Friedensforschung sei auf dem Weg von einer Wissenschaft »für den Frieden« zu einer Wissenschaft »über den Frieden« geworden (Bonacker 2011: 46-47).

Diese Trennung von Gegenstandsbereich und Wertbezug, die auch im Beitrag von Tanja Brühl (2012, in diesem Heft) anklingt, halte ich für problematisch. Was unter »Frieden« zu verstehen ist, ist von individuellen oder gesellschaftlichen Set-

1 Wobei die Terminologie keinesfalls einheitlich ist. Manche »peace studies«-Institute wie das Joan B. Kroc Institute an der University of Notre Dame sind eher der Friedensforschung zuzurechnen.

2 Ein - zugegeben subjektiver - Indikator dafür ist die Teilnahme deutscher Friedensforscherinnen an den Tagungen der International Peace Research Association (IPRA) einerseits und denen der International Studies Association (ISA) andererseits. Während die Teilnahme an IPRA-Konferenzen seit den 1980er Jahren stark abgenommen hat, ist die an ISA-Konferenzen in der jüngeren Vergangenheit deutlich gestiegen. Die IPRA-Tagungen werden von Vertretern aus den Friedensstudien dominiert, während ISA-Tagungen auf in der Regel höherem akademischem Niveau einem breiten Spektrum von Disziplinen und Forschungstradition ein Forum bieten. 
zungen abhängig. Die Entscheidung darüber, was als Forschung »über den Frieden« und damit als Friedensforschung angesehen werden kann, wird (auf nicht post-positivistischer Grundlage) nicht ohne (Vor-)Urteile dessen möglich, was »Frieden « ist. Und das ist - nicht nur, aber besonders - in der Friedensforschung bekanntlich hoch umstritten (Schlotter/Wisotzki 2011; Jaberg 2009). Nun lässt sich aus post-positivistischer Sicht argumentieren, Forschung »über den Frieden« könne wissenschaftlich nur Forschung über die Benutzung des Begriffs »Frieden« im politischen oder wissenschaftlichen Diskurs sein. Damit würde die Friedensforschung aber extrem eingeschränkt. Besonders drastisch, weil gegen null gehend, wird diese Bestimmung des Gegenstands der Friedensforschung für die naturwissenschaftliche Friedensforschung. Sie wäre in post-positivistischer Sicht nur dann Friedensforschung, wenn sie sich etwa mit der Frage beschäftigen würde, wie es kommt, dass bestimmte Technologien vom Militär aufgenommen werden, andere aber nicht. Beides sind keine naturwissenschaftlichen Fragen.

Die IB hat hier weniger Probleme. Forschung »über« die internationalen Beziehungen, so wie sie von Akteuren beschrieben werden, kann sich mit sehr vielen Themen beschäftigen - und die IB hat das in den letzten Jahren in vielen Projekten und Arbeiten auch erfolgreich gemacht. Dazu gehören auch Fragen von Krieg und Frieden, Sicherheit und Unsicherheit. Von den Themen her lässt sich Friedensforschung als ein Unterfeld der IB bezeichnen, mit dem Unterschied, dass die Forschung in den IB überwiegend ohne explizite normative Setzung betrieben wird (siehe Tabelle 1). IB ist zwar für viele epistemologische Ansätze offen, allerdings werden Herangehensweisen, die den Wertbezug möglichst stark von der Forschung isolieren, bevorzugt (Sprintz/Wolisky-Nahmias 2004: 1-2).

Nur am Rande sei hier vermerkt, dass sich die genannten Unterschiede zwischen Friedensforschung und IB auch in einem benachbarten, teilweise überlappenden Gegenstandsbereich widerspiegeln. Die intensive Beschäftigung mit »Sicherheit« hat sich in den letzten Jahren so stark differenziert, dass sich inzwischen eigenständige Forschungstraditionen herausgebildet haben. Die Critical Security Studies haben viele Gemeinsamkeiten mit der Friedensforschung in ihren Anfängen. Zumindest für einen der dominanten Stränge (»Aberystwhyth School«) ist die kritische Theorie erkenntnistheoretischer Maßstab (Krause/Williams 1997; Buzan/Hansen 2009). Die Critical Security Studies haben sich aus der Kritik an einem Mainstream - in diesem Fall der Umformulierung und Erweiterung des Sicherheitsbegriffs aus dem Strategischen und später Sicherheitsstudien (Security Studies) entwickelt (siehe Tabelle 1). Sowohl die Security Studies als auch die Critical Security Studies sind in den IB institutionell in der Regel eng verbunden, wenn auch - wie in der frühen Friedensforschung - der Einfluss der Soziologie deutlich stärker ist als in der gegenwärtigen Friedensforschung (Dunn-Cavelty/Mauer 2010).

Im Ergebnis bleibt »Frieden« als Gegenstandsbereich der Friedensforschung von individuellen und kollektiven Bewertungen abhängig. Individuell für die einzelne Forscherin, die sich in dieser Forschungstradition verankern will, kollektiv in Institutionen der Friedensforschung, wie etwa der Deutschen Stiftung Friedensforschung (DSF). 
Eine der Fragen an Gutachter für Anträge an die DSF betrifft die Relevanz eines vorgeschlagenen Forschungsthemas für die Friedensforschung. In der Regel sind die Gutachter, die vor allem aufgrund ihrer fachlichen und thematischen Kompetenz angefragt werden, bereit, ein weites Spektrum an Gegenständen für förderfähig zu erklären. Im Stiftungsrat der DSF wird nach meiner Erfahrung häufiger danach gefragt und kontrovers diskutiert, ob eine Fragestellung zum Förderspektrum einer Stiftung für Friedensforschung gehört. Beispiele hierfür sind etwa Themen aus dem Umfeld der Armutsforschung oder der philosophischen Grundlagenforschung.

Gravierender für eine Eingrenzung von Friedensforschung auf »Forschung über Frieden « ist allerdings, dass nicht nur die Friedensforschung zu Fragen von Konflikt, Krieg und Frieden forscht. Die zentrale Unterscheidung zu den Strategischen Studien und den Sicherheitsstudien ist das Erkenntnisinteresse der kurz- und langfristigen Minderung von Gewaltanwendung in der Friedensforschung gegenüber dem Ziel der Verbesserung der Position der »eigenen Seite« gegenüber Bedrohungen und Risiken (siehe auch Tabelle 1). Friedensforschung und Strategische Studien mögen sich analytisch mit denselben Gegenständen befassen, etwa den Konsequenzen aus der Vermutung, dass der Klimawandel in Zukunft Sicherheitsprobleme schaffen könnte, unterscheiden sich aber grundsätzlich in Bezug auf die politischen Schlussfolgerungen (vgl. etwa Brzoska/Fischer 2011 mit Mazo 2010).

Über den engen Bereich post-positivistischer Forschung hinaus ist daher der Normorientierung der Friedensforschung nicht zu entkommen: Friedensforschung definiert sich als Forschung über den Frieden für den Frieden. Es bleibt aber die Frage, wie weit die Wertorientierung gehen sollte.

Dies ist, wie bereits angedeutet, in der Vergangenheit in den verschiedenen erkenntnistheoretischen Strängen der Friedensforschung, wie in den Sozialwissenschaften insgesamt, durchaus unterschiedlich beantwortet worden, mit den beiden Gegenpolen des kritischen Rationalismus einerseits und der kritischen Theorie andererseits (Jutila et al. 2008). Anfang der 1970er Jahre verdrängten die kritischen Friedensforscherinnen an deutschen Universitäten mit einem Verständnis von Normativität, das nicht nur die Wahl des Gegenstandsbereichs, sondern auch ein emanzipatorisches Erkenntnisinteresse umfasste, die Gründergeneration der Friedensforscherinnen, die eher dem kritischen Rationalismus verpflichtet waren, und danach mit der Friedensforschung überwiegend nichts mehr zu tun haben wollten (Wasmuth 1998). Diese Entwicklung fand aber von Ausnahmen abgesehen nur in Deutschland und seinen unmittelbaren Nachbarländern statt. Spätestens seit den 1980er Jahren begann darüber hinaus eine kritische Reflexion über den Anspruch der kritischen Theorie, also über die Voraussetzungen und Folgen eines normativen Erkenntnisinteresses für die Friedensforschung (Jaberg 2011; Bonacker 2011: 46-78).

Mit der verstärkten Reflexion der Wertgebundenheit der eigenen Forschungsarbeit sind Divergenzen innerhalb der Friedensforschung stärker hervorgetreten als dies in den Jahrzehnten davor der Fall war. Die heftigen Debatten über die Kriege im früheren Jugoslawien und insbesondere das externe Eingreifen mit militärischen Mitteln sind Beispiele dafür. Aktuell trifft dies für die Diskussion über die »respon- 
sibility to protect« zu, in der Friedensforscher - Beispiel Libyen - sowohl aufseiten der Skeptiker wie Befürworter zu finden sind. ${ }^{3}$

Derartige Debatten sind notwendig und in der Regel produktiv. Unterschiedliche Positionen sind Ausdruck sowohl unterschiedlicher Einschätzungen empirischer Befunde - Beispiel Libyen: Hat die NATO-Intervention mehr Leben gerettet als gekostet? - als auch unterschiedlicher Wertordnungen - im Falle Libyens etwa zur Berechtigung von Gewaltanwendung zur Gewaltminderung. Auch wenn Positionen »lediglich « Wertorientierungen widerspiegeln, sind sie damit nicht »unwissenschaftlich«. Nehmen wir etwa an, dass sich empirisch belegen ließe, dass die Libyen-Intervention von NATO-Staaten und Verbündeten mehr Leben gerettet als gekostet hat, ließe sich trotzdem vertreten, dass ein Verzicht auf diese Gewaltanwendung vorzuziehen gewesen wäre, etwa weil damit einer generellen (Re-)Legitimation von Gewaltanwendung durch »koloniale« Staaten im internationalen System Vorschub geleistet werde (zu den Grundlagen einer solchen Argumentation vgl. Jabri 2007).

Wissenschaftlich nicht akzeptabel ist das Bestehen auf empirischen Behauptungen, um damit normative Positionen zu bestätigen, auch wenn diese nicht haltbar sind. Diese Aussage ist allerdings insofern wenig hilfreich, als empirische Befunde fast immer umstritten sind. Das gilt in verstärktem Maße für die Gewichtung komplexer Tatbestände, die aus mehreren empirischen Elementen bestehen.

Ein Beispiel hierfür ist die Debatte um den Zusammenhang zwischen sich abzeichnendem Klimawandel und bewaffneten Konflikten. Nicht nur im politischen Feld, auch in der Wissenschaft werden hierzu starke Aussagen gemacht. So schrieben die Autorinnen des Wissenschaftlichen Beirats Globale Umweltfragen (WBGU), dass ohne entschiedenes Gegensteuern der Klimawandel

»zunehmend Spaltungs- und Konfliktlinien in der internationalen Politik hervorrufen [wird], weil er vielfältige Verteilungskonflikte in und zwischen Ländern auslöst, um Wasser, um Land, um die Bewältigung von Flüchtlingsbewegungen und um Kompensationszahlungen« (WBGU 2007: 1).

Eine solche Aussage lässt sich mit dem gegenwärtigen Forschungsstand nicht als empirisch gesichert ansehen, im Gegenteil, die Mehrheit der vorliegenden Studien findet keinen Zusammenhang zwischen Wetterveränderungen und bewaffneten Konflikten im letzten halben Jahrhundert (Saleyhian 2008: 324, 326; Brzoska 2009: 137-138). Gleichzeitig aber kann mit gängigen Theorien über die Faktoren, die das globale Niveau bewaffneter Konflikte beeinflussen, prognostiziert werden, dass der Klimawandel zu einer Erhöhung der Zahl und Intensität bewaffneter Konflikte führen kann, etwa weil es vermehrt zu Auseinandersetzungen über knappe Ressourcen kommt oder weil politische Institutionen überlastet werden (WBGU 2007: 7-8). Dass sich dieses Muster empirisch bisher bestenfalls in Einzelfällen - Darfur ${ }^{4}$ - gezeigt hat, könnte daran liegen, dass der Klimawandel gerade erst begonnen hat. In-

3 Symptomatisch dafür ist die Einleitung zum Friedensgutachten 2011 (Johannsen et al. 2011: 20-24). Die Herausgeber konnten sich nicht auf eine Einschätzung einigen und lieferten daher neben einer gemeinsamen deskriptiven Analyse mehrere Schlussfolgerungen.

4 Auch der Fall Darfur ist umstritten (vgl. Schreiber 2011). 
sofern lässt sich durchaus auf wissenschaftlicher Basis behaupten, dass die Vermeidung von Klimawandel die zukünftige Zahl und Intensität bewaffneter Konflikte vermindern würde, allerdings nicht ohne den Hinweis darauf, dass dies eine nicht empirisch gesicherte Prognose darstellt. Eine weitergehende Behauptung ist angesichts der Literaturlage nicht akzeptabel, auch wenn sie in Bezug auf Vermeidung des Klimawandels in »bester« Absicht geschieht.

\section{Praxisorientierung}

Normativität ist im Übrigen nach meiner Erfahrung weniger ein Problem einer selbstkritischen Friedensforschung als der öffentlichen Erwartungen an die Friedensforschung. Das zeigt sich zum Beispiel an Stellungnahmen aus der Friedensforschung, die nicht dem vorgegebenen Bild der Ergebnisse der Friedensforschung entsprechen. Um das gewählte Beispiel wieder aufzugreifen: Das Publikum erwartet Stellungnahmen aus der Friedensforschung, in denen eine zunehmende Kriegsgefahr durch den Klimawandel konstatiert wird. Abweichende Positionen führen zu Irritationen. Das beschwört die Gefahr strategischen Verhaltens, um bei den als relevant angesehenen gesellschaftlichen Akteuren Gehör zu finden. Oder es führt da$\mathrm{zu}$, von diesen nicht mehr wahrgenommen zu werden. Angeblich von Karl Deutsch stammt das Bonmot, nachdem das Verhältnis der Politik zur Wissenschaft wie das des Betrunkenen zum Laternenpfahl sei: Sie suchen Halt und nicht Erleuchtung.

Obwohl kein spezifisches Problem der Friedensforschung, ist das WissenschaftPraxis-Verhältnis aufgrund der Forderung nach Praxisorientierung für die Friedensforschung von besonderem Gewicht. Die Hoffnung der Wissenschaftlerin ist, dass die Qualität der eigenen Forschung das Interesse gesellschaftlicher und politischer Akteure an bestimmten Erkenntnissen aussticht. Das ist der Idealfall, der Normalfall ist allerdings der Balanceakt zwischen Anpassung und Provokation, oder wie Herbert Wulf es formuliert hat, das »Minenfeld« der Erwartungen (Wulf 2011: 499).

Praxisorientierung wird für die und in der Friedensforschung generell als konstitutiv angesehen und ist wichtiges Unterscheidungsmerkmal von den IB. Sie ist auch eine wichtige Legitimation für die Förderung von Friedensforschung durch öffentliche Geldgeber, etwa die DSF. Anlässlich der Gründung im Jahre 2001 erklärte die damalige Bundesministerin und starke Förderin der DSF, Edelgard Buhlmahn: »Die Deutsche Stiftung Friedensforschung soll ein neues Instrument der Politikberatung sein und die Bundesregierung bei der Krisenprävention und der Konfliktbeilegung unterstützen« (BMBF 2001).

Auch für die Praxisorientierung gilt, dass in den Anfangsjahren der deutschen Friedensforschung in den späten 1960er und frühen 1970ern scharfe Debatten über Grundsatzfragen geführt wurden. Während die Gründergeneration, die überwiegend aus der traditionellen IB kam, die Friedensforschung in der Tradition des Popperschen »piecemeal social engineering« in den Dienst einer aufgeklärten deutschen Außen- und Sicherheitspolitik stellen wollte, forderten die Vertreter der kriti- 
schen Friedensforschung eine grundsätzliche Infragestellung der herrschenden Politik. Ihr Praxisstandpunkt war der der kritischen Theorie (Senghaas 1972: 28-93).

Christoph Meyer (2012, in diesem Heft) kritisiert in seinem Beitrag zu diesem Forum die Überbetonung des analytischen Elements in der Literatur zu Frühwarnung aus der Friedensforschung und fordert, mehr intellektuelle Energie in qualitative Analysen und das genaue Verständnis der Konsumenten und Geldgeber zu investieren. Er stellt sich damit in die Tradition des "piecemeal social engineering « und folgt, wie das Zitat von Edelgard Buhlmahn zeigt, den Erwartungen der politischen Entscheidungsträger. Das erste Problem mit dieser Position ist wissenschaftsimmanent. Der Wissensstand über Dynamik und Konsistenz von Verursachungsketten für bewaffnete Konflikte ist zu gering, um Vorhersagen über die Wahrscheinlichkeit von deren Auftreten mit der Präzision machen zu können, die Akteure im Bereich der Frühwarnung gerne hätten (Ward et al. 2010). Die Erfinder und Betreiber von Frühwarnsystemen sollten dies ihren Klienten deutlich machen und tun dies in der Regel auch. Das zweite Problem ist die Orientierung auf Entscheidungsträger mit bestimmten Erwartungen. Dies erfordert eine strategische Analyse der Interessen dieser Akteure an »earlyaction « - mit der Gefahr strategischen Verhaltens oder des Unmuts der gewünschten Adressaten. Womit wir wieder im bereits angesprochenen Minenfeld der Erwartungen wären. Friedensforschung nur darauf beschränken zu wollen, scheint mir auch noch aus einem dritten Grund problematisch. Frühwarnung ohne politisches Kalkül muss nicht unpolitisch sein. In der "Gesellschaftswelt« können gerade solche Warnungen besonderes Gehör finden, die von der politischen Klasse, aus was für Gründen auch immer, ignoriert werden.

\section{Interdisziplinarität}

Friedensforschung ist keine eigenständige wissenschaftliche Disziplin, mit - wie auch immer diffusen - Grenzen zu anderen Disziplinen. Sie weist zwar einige der oft für wissenschaftliche Disziplinen als typisch genannte Elemente, wie abgegrenzten Gegenstandsbereich, anerkanntes Repertoire an Konzepten und Theorien sowie als gesichert angesehenen spezifischen Wissensbestand auf (Krishnan 2009). Aber die Grundidee der Friedensforschung, alle interessanten und relevanten wissenschaftlichen Traditionen für die Erforschung von Frieden und Gewaltkonflikt und deren Bedingungen für gemeinsame Anstrengungen nutzbar zu machen, spricht gegen Versuche, auch das zentrale Element der institutionellen Abgrenzung anzustreben. Denn erfahrungsgemäß führt dies im Wettbewerb der Disziplinen zur Abgrenzung gegenüber anderen Fächern und erschwert die interdisziplinäre Zusammenarbeit. Die Offenheit zu vielen Fächern überwiegt die Nachteile, die ein Verzicht auf Disziplinierung mit sich bringt, wie etwa den Verzicht auf einen eigenen methodischen Zugang. Die Vorteile der Vielfalt illustrieren die verschiedenen Stu- 
diengänge im Umfeld der Friedensforschung mit ihren unterschiedlichen Überschneidungen mit etablierten wissenschaftlichen Disziplinen. ${ }^{5}$

Das schließt nicht aus, dass es eine dominierende Disziplin gibt, der nicht nur die Mehrheit der Friedensforschung Betreibenden zugehörig ist, sondern die auch eine Leitfunktion innehat. Diese Leitfunktion hat in der Geschichte der Friedensforschung über weite Strecken die Politikwissenschaft eingenommen. Das kann, wie Harald Müller (2012, in diesem Heft) in seinem Beitrag ausführt, nicht überraschen, sind doch Probleme des Friedens in der Regel politische Probleme, deren Regelung gesellschaftliche und politische Akteure erfordert. Nur in den späten 1960er und frühen 1970er Jahren war, vor allem aufgrund des starken Einflusses von Galtung, das Gewicht struktur-funktionalistischer Ansätze zumindest in Skandinavien dominierend und Friedensforscherinnen kamen überwiegend aus der Soziologie.

In den IB scheint mir das Bestreben nach disziplinärer Eigenständigkeit deutlich weiter verbreitet als in der Friedensforschung. Gelegentlich wird dabei daran gedacht, Masse und Schlagkraft der IB zu erhöhen, indem sie mit der Friedensforschung verbunden wird. Das macht insofern Sinn, als die Überlappung von IB und Friedensforschung nicht vollständig ist und sie sich, was erkenntnistheoretische Perspektiven und disziplinäre Ansätze angehen, gut ergänzen können. Das gilt besonders für Studiengänge. Allerdings kann sich die Friedensforschung nicht darauf beschränken (lassen), Ergänzungswissenschaft für die IB zu sein. Um ihrem Anspruch nach Forschung über und für den Frieden auf jeweils aktuellem Stand der Wissenschaft, wo auch immer sie disziplinär verortet ist, gerecht zu werden, muss sie sich offensiv um den Dialog und die Interaktion mit vielen Forschungstraditionen bemühen.

Inter- und transdisziplinärer Dialog und Interaktion ergeben sich selten von allein. Sie erfordern besondere intellektuelle und häufig auch bürokratische Anstrengungen. Sie sind, wie die Masterstudiengänge im Bereich der Friedensforschung zeigen, in der Lehre deutlich leichter zu organisieren als in der Forschung. Eine Konsequenz der Professionalisierung der Friedensforschung war die verstärkte Orientierung vor allem von Nachwuchswissenschaftlern an den bestehenden Disziplinen - weil diese einen etablierten Kanon an Theorien und Methoden boten und auch aus nachvollziehbaren Karrieregründen. Da dieselben Mechanismen auch in den anderen für die Friedensforschung interessanten Disziplinen und Forschungstraditionen wirken, gibt es von beiden Seiten, aus der Friedensforschung und anderen Wissenschaften, häufig Reserven bis hin zur Ablehnung transdisziplinärer Kooperation. Das trifft nach meiner Beobachtung neben den Naturwissenschaften vor allem für die Wirtschaftswissenschaften zu, nicht jedoch für die Rechtswissenschaft (Völkerrecht), die Geisteswissenschaften, die Psychologie oder die Pädagogik.

5 Wobei zumindest Grundzüge der IB in allen Studiengängen gelehrt werden. Für Grundinformationen zu den Studiengängen siehe http://www.afk-web.de/afk-home/infos-zu-studiengaengen.html. 
Die DSF hat durch besondere Förderung versucht, in den Naturwissenschaften die Voraussetzungen für solche Kooperation zu verstetigen. In Deutschland gibt es seit drei Jahrzehnten eine größere Gruppe, vor allem von Physikern, die Friedensforschung auf naturwissenschaftlicher Grundlage betreibt. Ihre Stellung in den etablierten Disziplinen bleibt allerdings trotz einiger Erfolge - so gibt es in der Deutschen Physikalischen Gesellschaft eine Arbeitsgruppe »Abrüstung und Rüstungskontrolle« - prekär. In der deutschen Wirtschaftswissenschaft ist das Interesse, mit der Friedensforschung zusammenzuarbeiten, bisher auf einzelne Personen beschränkt geblieben. Anders übrigens als in den USA und England, in denen es eine starke Tradition der Defense Economics aber auch einer kritischen ökonomischen Friedensforschung gibt. ${ }^{6}$

Aus Sicht der IB wird das Bemühen um Stärkung der Bedeutung anderer Disziplinen in der Friedensforschung gelegentlich skeptisch gesehen. So war die Strukturförderung der DSF in der Naturwissenschaft nicht unumstritten. Das ist nachvollziehbar, geht es ja auch um Fördergeld, das sozialwissenschaftlich ausgerichteter Forschung nicht zur Verfügung steht. Entscheidungen über die Fortführung derartiger Strukturförderung sollten vor allem auf der Grundlage einer Einschätzung getroffen werden, welchen zusätzlichen Beitrag für die Friedensforschung die Verbesserung der Dialogmöglichkeiten in anderen Disziplinen erbringen kann - im Vergleich mit der Förderung in der Friedensforschung etablierter Disziplinen und Forschungstraditionen.

\section{Schwerpunkte zukünftiger Forschung ${ }^{7}$}

Friedensforschung wird auch in Zukunft auf den gewaltförmigen oder gewaltträchtigen Großkonflikt als Gegenstand und dessen friedlichen Austrag als normative Orientierung fokussieren, als Forschung für den Frieden und über den Frieden. Das stellt die Forschung vor immer neue Herausforderungen, zum einen durch neue gewaltträchtige Konfliktdynamiken, wie die Veränderungen der globalen Machtarchitektur oder den Klimawandel, zum anderen werden aber auch neue Ideen und Instrumente des Umgangs mit Konflikten und zur Verhinderung der Gewalteskalation vorgeschlagen und entwickelt, deren Prüfung Aufgabe der Friedensforschung ist.

Zentrales Feld der Friedensforschung bleibt die Analyse organisierter Gewalt, vor allem aber nicht nur in der Form von bewaffneten Konflikten und deren Voraussetzungen, Verlauf, Bedingungen und Folgen, mit dem Ziel der Verminderung und Vermeidung von Gewaltkonflikten. Das schließt ausdrücklich auch die Forschung über Details von Kriegsführung und Kriegsvorbereitung ein. Anders als Christoph Meyer (2012) in seinem Beitrag sehe ich hier keine Scheuklappen in der

6 Ausdruck dafür sind etwa die Zeitschriften Economics of Peace and Security Journal, (http://www.epsjournal.org.uk/) und Peace Economics, Peace Science and Public Policy (http://www.bepress.com/peps/).

7 Die folgenden Ausführungen beruhen im Grundsatz auf Brzoska/Fischer (2011). 
Friedensforschung. Um nur zwei Beispiele zu nennen: Im Rahmen der Forschung zu den neuen Kriegen hat eine intensive Auseinandersetzung mit Veränderungen zu den Technologien und Taktiken von bewaffneten Gruppen stattgefunden (Brzoska 2004b); die Forschung zu den Möglichkeiten konventioneller Rüstungskontrolle und Abrüstung beschäftigt sich ausführlich mit Einsätzen und Einsatzszenarien moderner Waffen (Altmann et al. 2011).

Die Forschung zu organisierter Gewalt ist weit davon entfernt, darüber Einigkeit zu erzielen, welche Faktoren dafür verantwortlich sind, wann und wie Positionskonflikte über Interessen oder Werte gewaltförmig werden. Antworten auf Fragen nach der zukünftigen Entwicklung der Zahl und Intensität bewaffneter Konflikte, etwa aufgrund zunehmenden Klimawandels, sind somit Spekulationen auf der Grundlage nicht gesicherter Erkenntnisse. Gerade solche Fragen aber werden im politischen und gesellschaftlichen Feld aktuell dringlich gestellt. Besonderes Potenzial für Erkenntnisfortschritte sehe ich darin, die verschiedenen Forschungstraditionen innerhalb der Friedensforschung, die kaum miteinander kommunizieren, besser zu verzahnen. Das gilt zum einen für die quantitative und die qualitative Forschung und zum anderen für die Forschung zu bewaffneten Konflikten einerseits und zu anderen Formen der Gewalt - Terrorismus, Jugendgewalt, Gewaltaufständen - andererseits. Um das Beispiel Klimawandel und Konflikte noch einmal aufzugreifen: Fallstudien und makro-quantitative Forschung sind bis vor Kurzem mit durchaus unterschiedlichen Ergebnissen und nur geringem Bezug aufeinander vorangetrieben worden. Die Erweiterung der Analyse über bewaffnete Konflikte hinaus auf andere Gewaltformen beginnt gerade - und vermindert dabei das benannte Desiderat der Erkenntnisse zum Übergang von Positionskonflikten in Gewaltaustrag.

Ein anderes Thema von weiter großem Gewicht für die Friedensforschung ist die Friedenskonsolidierung in Nachkriegsgesellschaften. Die Krise des »liberal peace« erfordert eine kritische Analyse überkommener Theorien und Ansätze, die nicht zuletzt aus der Friedensforschung kamen. Besonderes Augenmerk sollten dabei Fragen des - militärischen und nicht-militärischen - Engagements externer Akteure haben. Solange keine grundlegend neue Theorie der Transformation von einer Kriegs- zu einer Friedensgesellschaft in Sicht ist, müssen weiter Teilaspekte untersucht werden - auf der Grundlage einer kritischen Sicht auf gängige liberale Theorien (Newmanet al. 2009; Kühn 2009).

Die Forschung über und für Frieden steht darüber hinaus vor neuen Herausforderungen. Die Ambivalenz von Globalisierung und Regionalisierung, von Auflösung von Grenzen und deren erneutem Aufbau ist bisher erst im Ansatz reflektiert und in Erklärungsansätze eingebaut. Die wirtschaftliche und politische Bedeutung Chinas, Indiens und mit Abstrichen auch Brasiliens, wächst. Die Vereinigten Staaten und Europa werden nicht auf Dauer internationale Organisationen und Normenbildung dominieren können, wie sie es seit dem Ende des Kalten Kriegs getan haben. Das wird absehbare Folgen für die internationale »governance« von Gewaltprävention und Friedenskonsolidierung haben. Die wachsende Knappheit an natürlichen Ressourcen, vor allem Ö1, könnte bestehende Konflikt-Regelungsmechanismen, hier 
vor allem den Weltmarkt, überlasten und aushebeln. Der Klimawandel könnte darüber hinaus als »threat multiplier« zur Eskalation bestehender Konflikte führen.

Die vorstehenden Sätze enthalten viele »kann« und wenige »wird«. Ob die genannten Entwicklungen eintreten werden, ist offen - weil wir es nicht wissen und weil die Entwicklungen durch gesellschaftliche und politische Akteure entscheidend beeinflusst werden können. Friedensforschung ist keine Zukunftsforschung und sollte es auch nicht sein. Sie arbeitet vorrangig mit Methoden und Theorien, die die Vergangenheit und bestenfalls noch die Gegenwart reflektieren und deren Projektionen in die Zukunft mit großen Unsicherheiten behaftet sind. Sie muss sich aber auf dieser Grundlage Zukunftsthemen nicht nur stellen, sondern diese auch suchen. Orientierung an Gewaltminderung und gesellschaftliche Relevanz bleiben dabei ihre Vorgaben, Wissenschaftlichkeit ihr Fundament. Die IB ist vor allem für Letzteres ein unverzichtbarer, aber kein exklusiver Partner.

\section{Literatur}

Altmann, Juergen/Kalinowski, Martin B./Kronfeld-Goharani, Ulrike/Liebert, Wolfgang/Neuneck, Götz 2011: Naturwissenschaft, Krieg und Frieden, in: Schlotter, Peter/Wisotzki, Sibylle (Hrsg.): Friedens- und Konfliktforschung - ein Studienbuch, Baden-Baden, 410-445.

Battke, Kathleen/Hauswedell, Corinna 1993: Handbuch Friedenswissenschaft. Expertinnen, Institutionen, Hochschulangebote, Literatur, Marburg.

BMBF (Bundesministerium für Bildung und Forschung) 2001: Pressemitteilung Nr. 57/2001 vom 27.4.2001, in: http://www.bmbf.de/_media/press/pm_20010427-057.pdf; 23.03.2012.

Bonacker, Thorsten 2011: Forschung für oder über den Frieden? Zum Selbstverständnis der Friedens- und Konfliktforschung, in: Schlotter, Peter/Wisotzki, Simone (Hrsg.): Friedens- und Konfliktforschung, Frankfurt a.M., 46-77.

Brzoska, Michael 2004a: Human Security - mehr als nur ein Schlagwort?, in: Weller, Christoph/Ratsch, Ulrich/Mutz, Reinhard/Schoch, Bruno/Hauswedell, Corinna (Hrsg.): Friedensgutachten 2004, Münster, 239-348.

Brzoska, Michael 2004b: »New Wars« Discourse in Germany, in: Journal of Peace Research 41:1, 107-117.

Brzoska, Michael 2009: The Securitzation of Climate Change and the Power of Conceptions of Security, in: Sicherheit und Frieden 27:3, 137-145.

Brzoska, Michael/Fischer, Martina 2011: Friedensforschung für das 21. Jahrhundert, in: Sicherheit und Frieden 29: 1, 53-57.

Brzoska, Michael/Kalinowski, Martin/Matthies, Volker/Meier, Berthold (Hrsg.) 2011: Klimawandel und Konflikte. Versicherheitlichung versus präventive Friedenspolitik?, BadenBaden.

Buzan, Barry/Hansen, Lene 2009: The Evolution of International Security Studies, Cambridge.

Czempiel, Ernst-Otto 1981: Internationale Politik. Ein Konfliktmodell, Paderborn.

Dietrich, Wolfgang/Alvarez, Josefina Echavarría/Esteva, Gustavo/Ingruber, Daniela/Koppensteiner, Norbert (Hrsg.) 2011: The Palgrave International Handbook of Peace Studies: A Cultural Perspective, London.

Dunn-Cavelty, Myriam/Mauer, Victor 2011: Routledge Handbook of Security Studies, London. 
Flemes, Daniel (Hrsg.) 2010: Regional Leadership in the Global System: Ideas, Interests and Strategies of Regional Powers, Burlington, VT.

Guzzini, Stefano/Jung, Dietrich 2004: Contemporary Security Analysis and Copenhagen Peace Research, London.

Hauswedell, Corinna 1997: Friedenswissenschaften im Kalten Krieg. Friedensforschung und friedenswissenschaftliche Initiativen in der Bundesrepublik in den achtziger Jahren, Baden-Baden.

Jaberg, Sabine 2009: Vom Unbehagen am Normverlust zum Unbehagen mit der Norm? Zu einem fundamentalen Problem der neueren Friedensforschung (Hamburger Beiträge zur Friedensforschung und Sicherheitspolitik 152), Hamburg.

Jaberg, Sabine 2011: Friedensforschung, in: Gießmann, Hans J./Rinke, Bernhard (Hrsg.): Handbuch Frieden, Wiesbaden, 53-70.

Jabri, Vivienne 2007: War and the Transformation of Global Politics, London.

Johannsen, Margret/Debiel, Tobias/Fröhlich, Christiane/Hauswedell, Corinna/Schoch, Bruno (Hrsg.) 2011: Stellungnahme der Herausgeberinnen und Herausgeber. Aktuelle Entwicklungen und Empfehlungen, in: Friedensgutachten 2011, Münster, 3-30.

Jutila, Matti/Pehkonen, Sahmu/Väyrynen, Tarja 2008: Resuscitating a Discipline: An Agenda for Critical Peace Research, in: Millennium 36: 3, 623-640.

Krause, Keith/Williams, Michael C.1997: Critical Security Studies: Concepts and Cases, London.

Kühn, Florian 2009: Sicherheit und Entwicklung in der Weltgesellschaft. Liberales Paradigma und Statebuilding in Afghanistan, Wiesbaden.

Krishnan, Armin 2009: What Are Academic Disciplines? Some Observations on the Disciplinarity vs. Interdisciplinarity Debate (ESRC National Center for Research Methods, NCRM Working Paper Series 03/09), Southampton.

Lopez, George 1999: Peace Studies: Past and Future, in: Annals of the American Association of Political Social Science 109: 1, 9-13.

Mazo, Jeffrey 2010: Climate Conflict (Adelphi Papers 409), London.

Meyer, Christoph 2012: Normative, theoretische und praxeologische Defizite der Friedensforschung am Beispiel der Konfliktprävention, in: Zeitschrift für Internationale Beziehungen 19: 1, 185-197.

Müller, Harald 2012: Über allen Gipfeln ist Ruh - Zum Verhältnis von Friedensforschung und IB, in: Zeitschrift für Internationale Beziehungen 19: 1, 155-169.

Newman, Edward/Richmond, Oliver P./Paris, Roland (Hrsg.) 2009: New Perspectives on Liberal Peacebuilding, Tokio.

Nicklas, Hans/Gantzel, Klaus Jürgen 1975: Außenpolitische Freund-Feind-Bilder in der Bundesrepublik Deutschland 1949-1971, Bonn.

Salehyan, Idean 2008: From Climate Change to Conflict: No Consensus yet, in: Journal of Peace Research 45: 3, 315-326.

Schlichte, Klaus 2012: Die Internationalen Beziehungen als Kirche, die Friedens- und Konfliktforschung als Sekte? Anmerkungen zur Disziplin, in: Zeitschrift für Internationale Beziehungen 19: 1, 143-154.

Schlotter, Peter/Wisotzki, Simone 2011: Stand der Friedens- und Konfliktforschung - Zur Einführung, in: Schlotter, Peter/Wisotzki, Simone: Friedens- und Konfliktforschung, Frankfurt a. M., 9-45.

Schreiber, Wolfgang 2011: Darfur - der erste Klimakrieg?, in: Brzoska, Michael/Kalinowski, Martin B./Matthies, Volker/Meyer, Berthold (Hrsg.): Klimawandel und Konflikte, Baden-Baden, 217-230.

Senghaas, Dieter 1972: Rüstung und Militarismus, Frankfurt a. M.

Sprintz, Detlev/Wolinski-Nahmias, Yael (Hrsg.) 2004: Models, Numbers and Cases: Methods for Studying International Relations, Ann Arbor, MI.

Ward, Michael D./Greenhill, Brian D./Bakke, Kristin M. 2010: The Perils of Policy by P-Value: Predicting Civil Conflicts, in: Journal of Peace Research 47: 3, 363-375. 
Wasmuht, Ulrike C. 1998: Geschichte der deutschen Friedensforschung. Entwicklung Selbstverständnis - Politischer Kontext, Münster.

WBGU (Wissenschaftlicher Beirat Globale Umweltfragen) 2007: Welt im Wandel. Sicherheitsrisiko Klimawandel, Berlin.

Wulf, Herbert 2011: Frieden und Politikberatung, in: Gießmann, Hans Joachim/Rinke, Bernhard: Handbuch Frieden, Wiesbaden, 495-503. 